\title{
The Effects Of Trunk Sensory Stimulation Training On Standing Balance Performance In The Elderly
}

\author{
Toshiaki Tanaka, Norio Kato, Tomoya Miyasaka, Yusuke Maeda, Takashi Izumi
}

\begin{abstract}
Purpose: Posture becomes unstable with aging due to decline in both motor and sensory function, resulting in increased risk of injury, hospitalization, or even mortality due to falls. The tactile sensation of the trunk will also be important in standing balance. The purpose of the study was to reveal the characteristics of vibratory sensation function in the lumbar region of the trunk in elderly people using a new vibration device that we developed, and to verify the effects of trunk tactile sensation training on standing balance performance in elderly people.

Methods: The elderly subjects were divided randomly into two groups, the control group and the lumbar training group, and general information including age and past history of falls was collected in each group. Lumbar region vibratory sensation training was carried out for 10 weeks. The subjects were elderly people aged 65 or over, who were divided into the training group and the control group for comparison and analysis. Balance was measured as static balance and dynamic balance, and vibratory sensation was evaluated as the rate of correct responses.

Results: In the results, the lumbar training group showed improvement over time in correct response rate. A strong correlation was found between lumbar vibratory sensation and dynamic balance performance, which is related to the causes of falls.

Conclusion: These results suggested that a trunk training may contribute to improved dynamic balance performance. It may be concluded that tactile sensation in the trunk is particularly important for avoiding falls when standing or walking.
\end{abstract}

Index Terms - trunk tactile sensation, sensory training, new vibratory device, dynamic standing balance ability, the elderly.

\section{INTRODUCTION}

Posture becomes unstable with aging due to decline in both motor and sensory function, resulting in increased risk of injury, hospitalization, or even mortality due to falls [1]. There has been a report that one third of all elderly people in the United States suffer falls every year [2], and in Japan the rate of falls among the elderly every year is reported to be over $20 \%$ [3]. In particular, fractures due to falls are a major factor in elderly people starting to need care, and gait and balance disorders accompanying decline in muscle strength are a risk factor for falls [1].

The most frequently used technique to evaluate the ability of static and dynamic postural stability, is the measurement of

Toshiaki Tanaka, Faculty of Health Sciences, Hokkaido University of Science, Sapporo, Japan. / The Research Center for Advanced Science and Technology/ Institute of Gerontology, The University of Tokyo, Tokyo, Japan

Norio Kato, Faculty of Health Sciences, Hokkaido University of Science, Sapporo, Japan

Yusuke Maeda, School of Health Sciences at Odawara, International University of Health and Welfare, Kanagawa, Japan the position and displacement of the center of pressure (COP) by using a force plate as a clinical assessment [4, 5]. Static balance is the ability to maintain postural stability and orientation with center of mass over the base of support and body at rest. Dynamic balance is the ability to maintain postural stability and orientation with center of mass over the base of support while the body parts are in motion [6].

Accurate sensory information from the soles of the feet is essential for control of standing balance [7]. In a prior study, we developed a device for evaluating vibratory sensation [8]. Using this device, we have shown that sensory function in the toes and the soles is lower in elderly people than in young people, and that sensation in the soles of the feet affects standing balance [9-11]. Other studies have reported that this tactile sensation of the legs is related to standing balance performance [12,13].

In addition to the soles of the feet, standing balance is also greatly affected by movement of the center of gravity, which is present in the trunk (in the region of the pelvis) when standing [14]. For this reason, there have been a number of studies focusing on the effects of trunk function on sitting and standing balance [15-17]. Just as foot sole sensation is an important element in standing balance, it may also be conjectured that tactile sensation of the trunk will also be important in standing balance. However, virtually no studies of the effects of tactile sensation of the trunk on standing balance can be found.

Balance training is used as a means to prevent falls in the elderly, and this is mainly an exercise intervention. A variety of intervention programs have been developed for muscle strength training mainly for the legs and the trunk, postural balance training, and gait training [18-21]. Thus, the most common approach to preventing falls is to maintain or improve balance performance through motor function training. However, there have been very few studies of methods to improve balance performance through training of the sensory function aspects, which are an important element in maintaining standing balance performance.

The present study therefore aims to reveal the characteristics of vibratory sensation function in the lumbar region of the trunk in elderly people using a new vibration device that we developed, and to verify the effects of trunk tactile sensation training on standing balance performance in elderly people.

\section{MATHODS}

\section{A. Subjects}

The subjects were 15 elderly people. The selection 


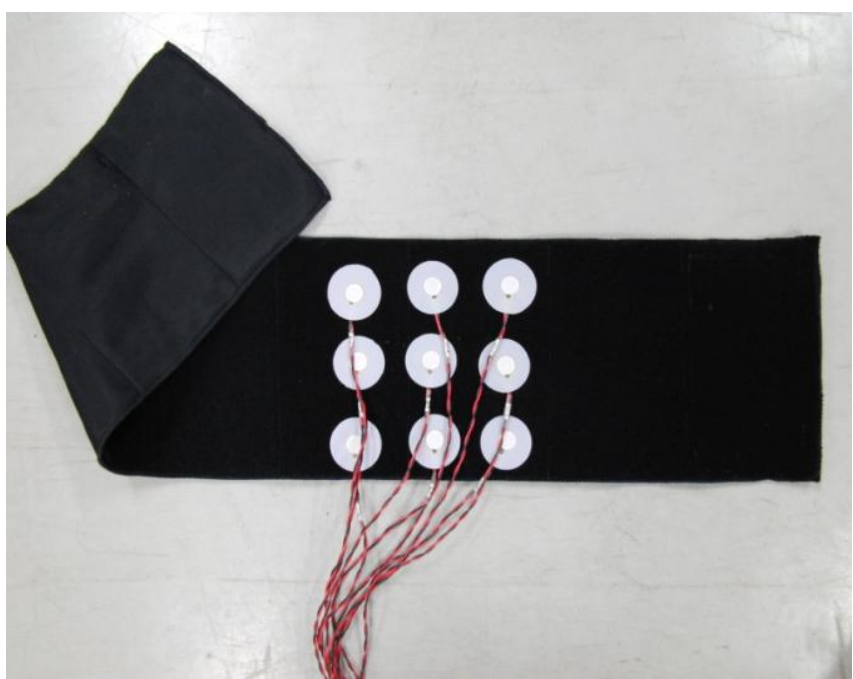

Fig. 1 Placement of lumbar region vibrators in the belt.

standards for the participants were those who had not fallen over during the previous year, those who had not suffered serious pain and musculoskeletal or neurological damage, those who were not on medication that might impair balance.

The subjects were divided randomly into two groups, the control group and the lumbar training group, and general information including age and past history of falls was collected in each group. The control group comprised eight subjects (mean age 67.0 years) and the lumbar training group comprised seven subjects (mean age 70.2 years). Vibratory sensation in the lumbar region was evaluated in both groups using the vibratory stimulation device that we developed. In addition, standing balance was evaluated using a force plate (Kyowa Electronic Instruments Co., Ltd.). To evaluate the effects of vibratory sensation training in the lumbar region on standing balance, a training period (10 weeks) was established for the lumbar training group, and after completion of the training, vibratory sensation and standing balance were evaluated in the same way as in the initial evaluation.

This study was performed in compliance with the ethical principles of the Declaration of Helsinki. The subjects agreed to participate in the study after receiving explanations of the purpose and procedures of the experiment, and they signed an informed consent statement before participation. The protocol for this study was approved by the ethics committee of the University of Tokyo (No.15-248).

\section{B. Evaluation of lumbar region vibratory sensation}

Vibratory sensation in the lumbar region was evaluated using the vibratory stimulation device that we developed. The vibrators used in the vibratory stimulation device were small vibration motors (Kyowa Electronic Instruments Co., Ltd.) of a type commonly used in mobile telephones. The voltage of the vibrator used in this study was equal to or less than $3 \mathrm{~V}$. The vibrator device never actually touched the skin of the subjects directly. The system used a one-chip microcomputer (PIC16F84A) to control the eight vibrators. The system can produce mechanical vibrations up to $100 \mathrm{~Hz}$. The nine vibrators were placed on a belt worn around the waist. The time for the stimulus to travel to all points was limited to $1 \mathrm{~s}$ in all patterns.

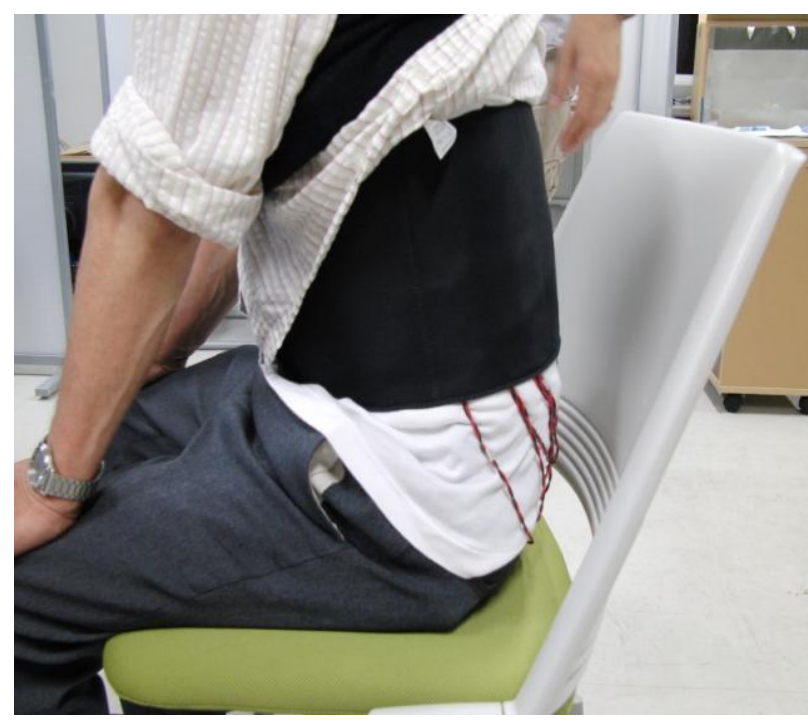

Fig. 2 Sensory evaluation in the lumbar region.

Vibratory stimulation was delivered to the lumbar region by means of a belt worn around the lumbar region which was fitted with nine vibrators arranged in a pattern $15 \mathrm{~cm}$ vertically x $20 \mathrm{~cm}$ horizontally, and subjects were instructed to respond whether or not there was any vibratory stimulation (Fig.1, 2). For a single trial, subjects were presented with 35 vibratory stimuli, and one trial was carried out on each of the left, center, and right of the trunk, a total of 105 stimuli. There were three patterns of vibration: stimulation in zero sites, stimulation in one site, and simultaneous stimulation in two sites, and these were presented randomly. In addition, for simultaneous stimulation in two sites, the two vibrators were placed vertically, horizontally, and diagonally (Fig.1). Subjects were instructed to respond whether the stimulation was given in "zero sites," "one site," or "two sites." To avoid any visual or auditory influence, subjects were seated and wore an eye mask and headphones for the evaluation.

\section{Evaluation of standing balance using a force plate}

For evaluation of static balance, subjects stood on a force plate (Kyowa Electronic Instruments Co., Ltd.) for $30 \mathrm{~s}$. The device used to evaluate standing balance was a force plate (Kyowa Electronic Instruments Co., Ltd.), which measured body sway parameters. The force plate contained four transducers, one in each corner of the force plate, to measure vertical forces indicating the postural sway. The signal from the transducers was amplified and then digitized by a computer. The computer program provided several indices of body sway calculated from the center of pressure. The chief body sway indices were area within the sway path $\left(\right.$ area, $\mathrm{mm}^{2}$ ) of the left-right and anterior-posterior co-ordinates of the center of pressure. The accuracy and hysteresis of the force plate were $\pm 1 \%$ and $\pm 0.1 \%$, respectively. The sampling rate of the force plate was $100 \mathrm{~Hz}$.

Sway of the center of gravity was calculated from the force plate data by multiplying the distance of antero-posterior travel of the center of gravity by the distance of left-right travel of the center of gravity, to give a rectangular area $\left(\mathrm{mm}^{2}\right)$ which was used for analysis. For evaluation of dynamic balance, the limits of stability (LoS) in the forward, 


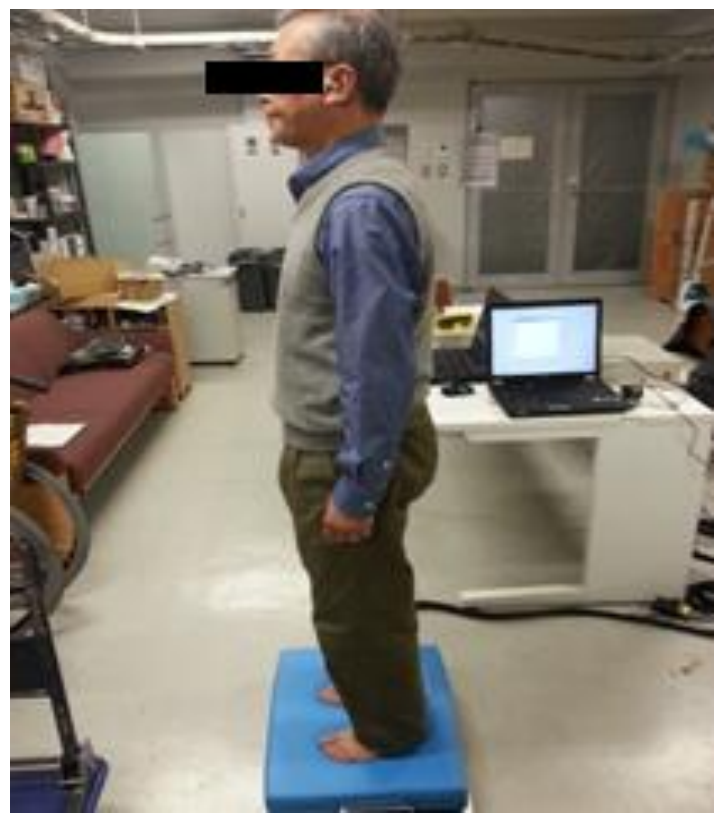

Fig.3 Evaluation of balance performance

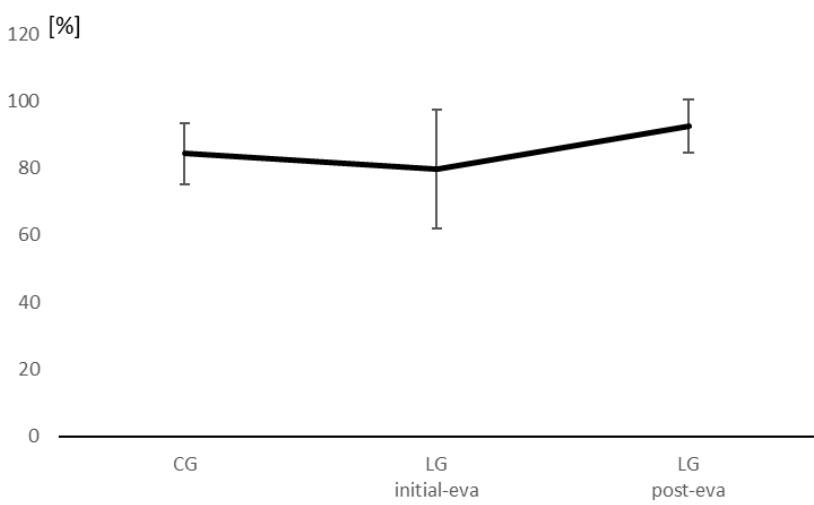

Fig.4 The mean rate of correct answer of vibratory sensation at the lumbar portion by a vibratory stimulation with one site. LG: The lumbar training group, CG: The control group, initial eva: The initial evaluation, post eva: The post-training evaluation

backward, leftward, and rightward directions were measured [22]. For this test, the maximum displacement of the center of gravity in the forward, backward, leftward, and rightward directions that could be maintained without changing the foot position while standing upright with the knees extended was measured. This test was performed with subjects standing barefoot with the arms at the side of the body, while looking at a marker placed $1.2 \mathrm{~m}$ to the front (Fig.3).

\section{Details of the lumbar training}

The lumbar training group was given stimulation training for lumbar region sensation for 10 weeks after the initial evaluations. Following the training period, the lumbar training group was again subjected to the evaluations outlined in II-2 and II-3 above. Lumbar training was carried out in the lumbar training group by delivering vibratory stimulation to the back of the trunk in order to train sensation to vibratory stimulation. The training was carried out with two vibratory sensation test tuning forks $(128 \mathrm{~Hz})$. Using the tuning forks, a researcher randomly applied vibratory stimulation to one or two of a total of 20 sites, each site $5 \mathrm{H} \mathrm{x} 5 \mathrm{~W} \mathrm{~cm}$, arranged five horizontally by four vertically on the

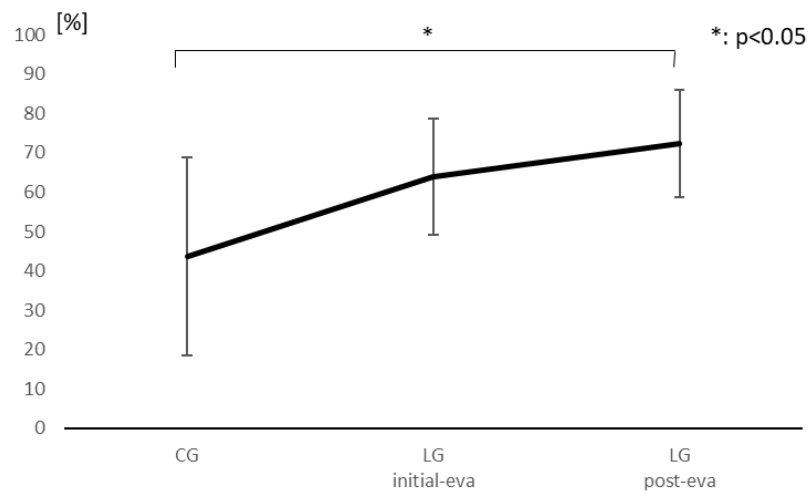

Fig.5 The mean rate of correct answer of vibratory sensation at the lumbar portion by a vibratory stimulation with two sites to vertical direction.

LG: The lumbar training group, CG: The control group, initial eva: The initial evaluation, post eva: The post-training evaluation

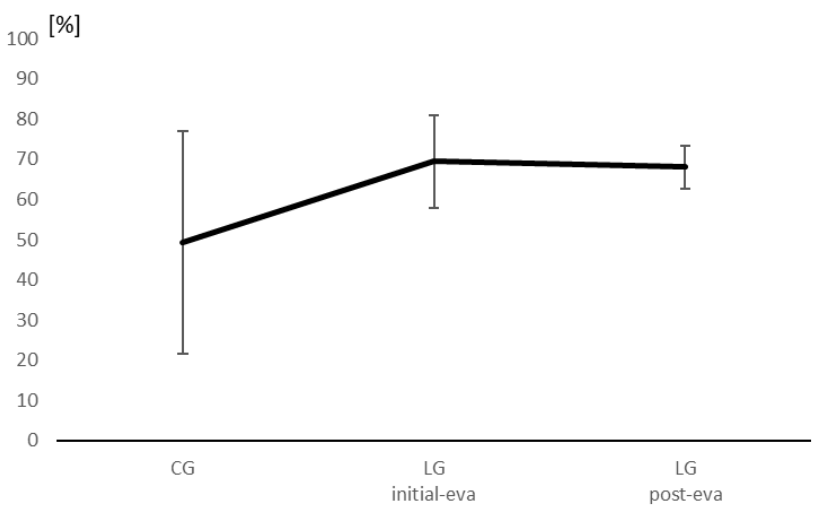

Fig.6 The mean rate of correct answer of vibratory sensation at the lumbar portion by a vibratory stimulation with two sites to horizontal direction.

LG: The lumbar training group, CG: The control group, initial eva: The initial evaluation, post eva: The post-training evaluation

lumbar region of the subject, and the subject responded when they felt the stimulation. The training was carried out for 15 min at a time, three or more times a week.

\section{E. Statistical analysis}

The items for analysis from the force plate were the area of sway of the center of gravity and the data from four directions in the LoS test, and the items for analysis from the sensory test were the responses from stimulation of one site and stimulation of two sites (arranged vertically, diagonally, and horizontally). For statistical analysis, the t-test and the Mann-Whitney test were used to compare the evaluation data of the control group and the lumbar training group (initial evaluation), and the control group and the lumbar training group after completion of training. In addition, initial evaluation and post-training evaluation data for the training group were compared by means of a paired t-test and the Wilcoxon signed-rank test. The level of significance was set at $5 \%$.

In addition, a multiple regression analysis was carried out with dynamic center of gravity sway data (seven values from 


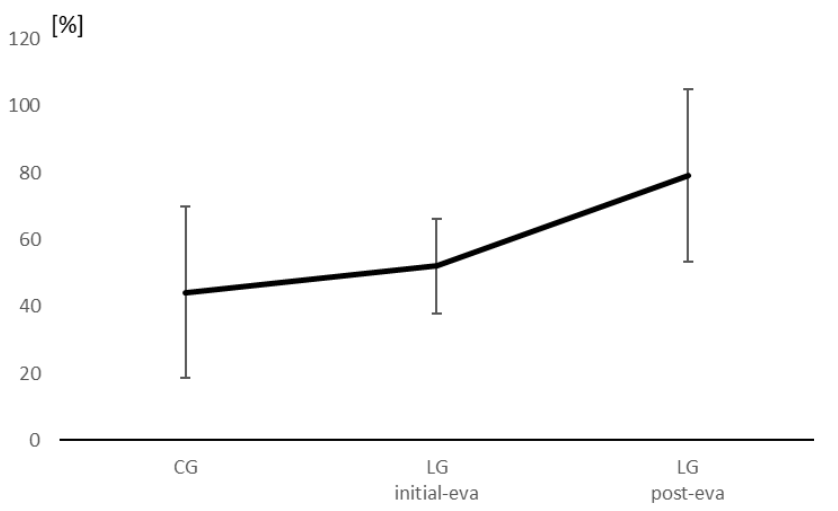

Fig.7 The mean rate of correct answer of vibratory sensation at the lumbar portion by a vibratory stimulation with two sites to diagonal direction.

LG: The lumbar training group, CG: The control group, initial eva: The initial evaluation, post eva: The post-training evaluation

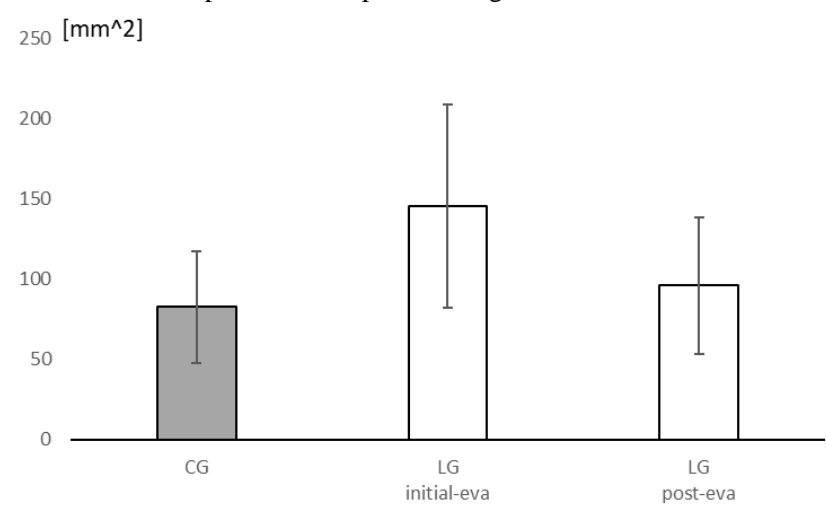

Fig. 8 The mean area of body sway static standing balance by using the force plate.

LG: The lumbar training group, CG: The control group, initial eva: The initial evaluation, post eva: The post-training evaluation

the LoS test: value for each of the four directions, total value for the four directions, total of anterior-posterior values, and total of left-right values) as dependent variables, and static center of gravity sway data (root mean square of area) and rate of correct responses in sensory tests as independent variables. For rate of correct responses, the multiple regression analysis was performed with respect to correct responses for stimulation of one site, two sites (arranged vertically, diagonally, and horizontally), and zero sites, and the test overall. Selection of variables was carried out by means of the stepwise method. The level of significance was set at $5 \%$.

\section{RESULTS}

Participation of seven subjects in the lumbar region training was planned at the start of the study, but due to poor health only four subjects (mean age 71.0) were able to carry out the training.

\section{A. Vibratory sensation evaluation}

In the vibratory sensation evaluation of the lumbar region,

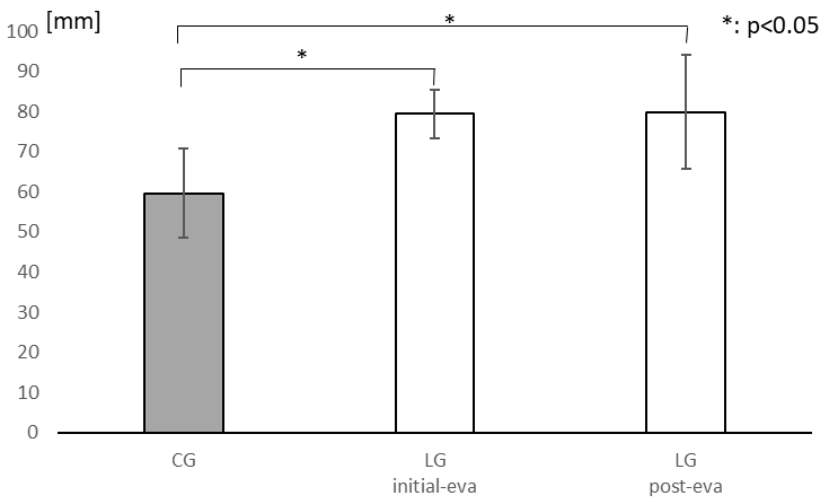

Fig.9 Mean maximum displacement values with the body inclined in forward direction when standing as an assessment of dynamic standing balance.

LG: The lumbar training group, CG: The control group, initial eva: The initial evaluation, post eva: The post-training evaluation

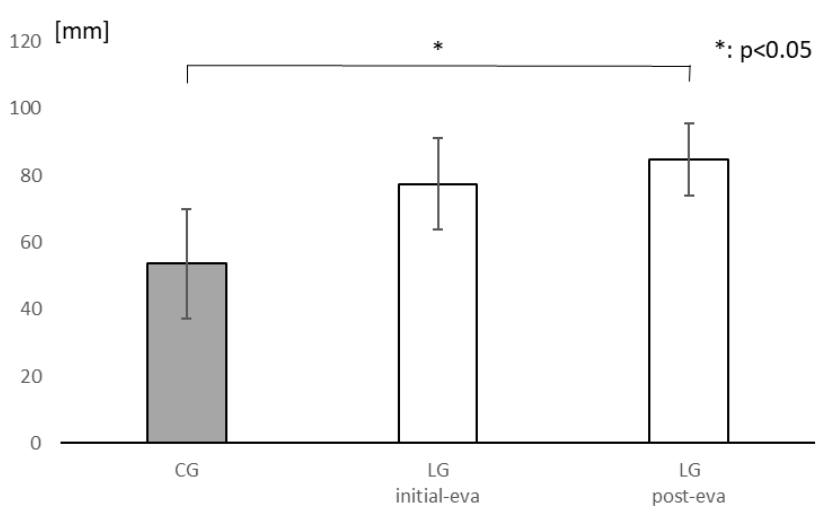

Fig.10 Mean maximum displacement values with the body inclined in backward direction when standing as an assessment of dynamic standing balance.

LG: The lumbar training group, CG: The control group, initial eva: The initial evaluation, post eva: The post-training evaluation

the results of the initial evaluation (first test) of the lumbar training group (four persons) showed that the mean rate of correct responses with one site stimulated was approx. $82 \%$ (Fig.4). When two sites were stimulated, the mean rate of correct responses was approx. $64 \%$ for two sites arranged vertically (Fig.5), approx. 69\% for two sites arranged horizontally (Fig.6), and approx. 52\% for two sites arranged diagonally (Fig.7). The results of the post-training evaluation (second test) of the lumbar training group showed that the mean rate of correct responses with one site stimulated was approx. 93\% (Fig.4). When two sites were stimulated, the mean rate of correct responses was approx. $72 \%$ for two sites arranged vertically (Fig.5), approx. 68\% for two sites arranged horizontally (Fig.6), and approx. 79\% for two sites arranged diagonally (Fig.7). In the control group (eight persons), the evaluation was only carried out once, and the results showed that the mean rate of correct responses with one site stimulated was approx. 84\% (Fig.4). When two sites were stimulated, the mean rate of correct responses was approx. 44\% for two sites arranged vertically (Fig.5), approx. $49 \%$ for two sites arranged horizontally (Fig.6), and approx. 


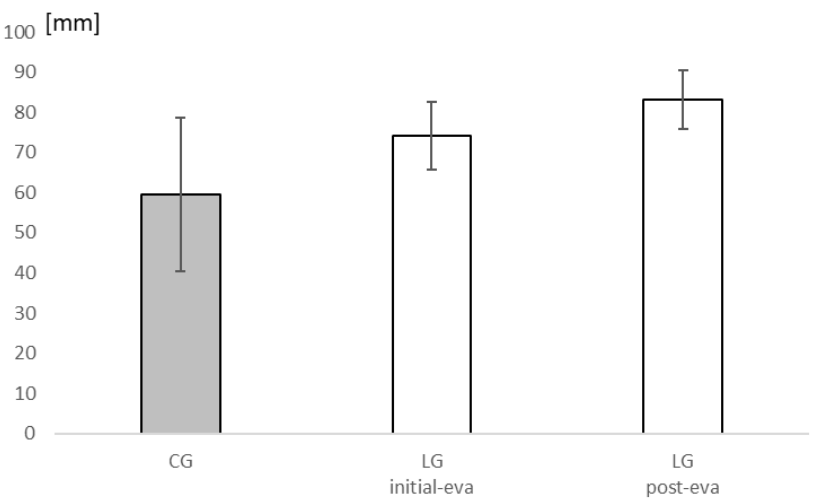

Fig.11 Mean maximum displacement values with the body inclined in rightward direction when standing as an assessment of dynamic standing balance.

LG: The lumbar training group, CG: The control group, initial eva: The initial evaluation, post eva: The post-training evaluation

44\% for two sites arranged diagonally (Fig.7).

Statistical analysis found no significant differences between the two groups or within the training group as a result of the trunk sensation training. However, the correct response rate when one lumbar site was stimulated rose to over $80 \%$ after training, an increase of 1.2 -fold with respect to the pre-training value. The correct response rate when two sites were stimulated was over $60 \%$, and with two sites arranged vertically there was a significant increase with respect to the control following training. With respect to the pre-training value, the post-training value showed a 1.1-fold increase with two sites arranged vertically and a 1.5-fold increase with two sites arranged diagonally. With two sites arranged horizontally there was no change, indicating no trend toward improvement.

\section{B. Evaluation of standing balance performance with a force plate}

\section{Static balance (standing still) performance evaluation (Fig.8)}

In the initial evaluation, the area of sway of the center of gravity was $82 \mathrm{~mm} 2$ in the control group and about $145 \mathrm{~mm} 2$ in the lumbar training group. In the post-training evaluation, the area of sway of the center of gravity was about $96 \mathrm{~mm} 2$ in the lumbar training group. Statistical analysis of the static balance data showed no significant differences between the control group and the lumbar training group, and no differences within the lumbar training group between the initial evaluation and the post-training evaluation.

\section{Dynamic balance performance evaluation}

Dynamic balance was evaluated from mean maximum displacement values with the body inclined in forward, backward, rightward, and leftward directions when standing. In the initial evaluation, the maximum displacement values in the control group were approx. $60 \mathrm{~mm}$ forward (Fig.9), 54 mm backward (Fig.10), $60 \mathrm{~mm}$ rightward (Fig.11), and 57 $\mathrm{mm}$ leftward (Fig.12). The maximum displacement values in the lumbar training group were approx. $79 \mathrm{~mm}$ forward (Fig.9), $78 \mathrm{~mm}$ backward (Fig.10), $74 \mathrm{~mm}$ rightward (Fig.11), and $88 \mathrm{~mm}$ leftward (Fig.12). In the post-training evaluation,

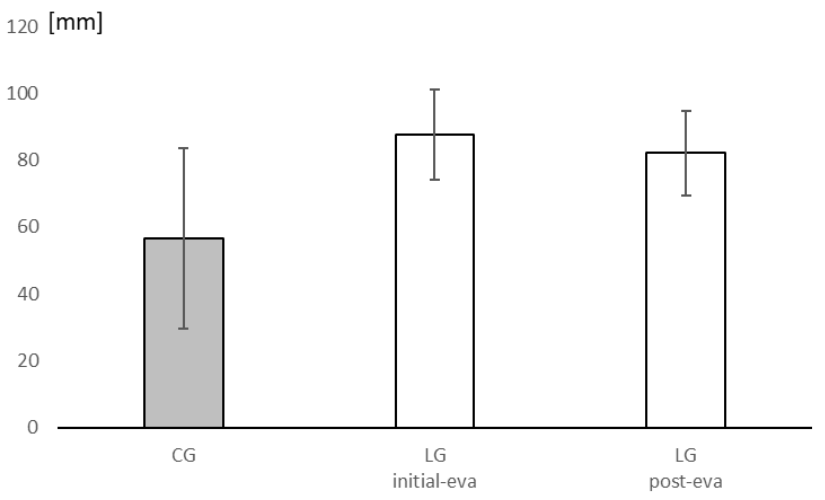

Fig.12 Mean maximum displacement values with the body inclined in leftward direction when standing as an assessment of dynamic standing balance.

LG: The lumbar training group, CG: The control group, initial eva: The initial evaluation, post eva: The post-training evaluation

the maximum displacement values in the lumbar training group were approx. $80 \mathrm{~mm}$ forward (Fig.9), $85 \mathrm{~mm}$ backward (Fig.10), $83 \mathrm{~mm}$ rightward (Fig.11), and $82 \mathrm{~mm}$ leftward (Fig.12). Statistical analysis of the dynamic balance data showed a significant difference in forward displacement between the control group and the lumbar training group at initial evaluation, and a significant difference in backward displacement between the control group and the lumbar training group at post-training evaluation.

The foregoing indicates that after 10 weeks of training, there was a significant difference with respect to the control group in some of the lumbar training group force plate data, and that overall, the lumbar training group showed a tendency toward improvement. Within the lumbar training group, the force plate data showed an overall tendency toward improvement following the training.

Furthermore, with the LoS test data of the lumbar training group taken as the dependent variable, the rate of correct responses in lumbar vibratory sensory tests showed a significant association as an independent variable. In particular, with total forward and backward limit of stability test value and rate of correct response for lumbar stimulation with two sites arranged vertically, significant simple regression equations were found at both initial evaluation and post-training evaluation, as follows.

Initial:

total antero-posterior $\operatorname{LoS}$ test value $=79.20+1.02 \mathrm{x}$ lumbar two sites vertically

$(\mathrm{p}<0.05)$ simple correlation coefficient $\mathrm{r}=0.566$

Post-training:

total antero-posterior LoS test value $=70.418+1.163 \mathrm{x}$ lumbar two sites vertically

$(p<0.05)$ simple correlation coefficient $r=0.567$

\section{DISCUSSIONS}

With the vibratory sensation training, there were no significant differences between the two groups and no significant differences pre- and post-training within the lumbar training group, but the correct response rate with stimulation of two sites was lower than with stimulation of one site. This is similar to the finding that there is a tendency in elderly people toward decrease in sensitivity to vibratory 
stimulation in threshold tests for vibratory stimulation delivered to the legs [8, 10, 23]. However, as a result of the training, the post-training evaluations showed a 1.1-fold increase with two sites arranged vertically and a 1.5 -fold increase with two sites arranged diagonally with respect to the pre-training values. There was a trend with the vibratory sensation stimulus training for a greater increase in the correct response rate with stimulation of two sites than the correct response rate with stimulation of a single site. The results show that vertical and diagonal pairs of stimuli are more readily sensed than horizontal pairs of stimuli, which is the same as the results reported for touch-pressure sensation tests with the fingers $[24,25]$. The improvement in trunk tactile sensation function shown by the increase in correct response rate for two sites may perhaps be important in postural control. In particular, it has been reported that simultaneous stimulation of two sites requires more complex processing than single stimulation of one site, and hence is more difficult for elderly people to perceive [26, 27].

The mechanoreceptors that react to simultaneous stimulation of two sites are likely to affect spatial resolution and postural changes. Improvement in sensing and reacting to stimulation of two sites may therefore allow a person to perceive movements of the center of gravity and thus to control posture, meaning that trunk sensory training may be important for postural control.

In the investigation of standing balance, no significant differences in static balance were seen between the groups. In the LoS test, which is a test of dynamic balance, forward and backward displacement in the training group showed significant increase with respect to the control group after 10 weeks of training. This suggests that trunk training may contribute to improved dynamic balance performance. Stimulation for the sensory training was delivered by means of randomly shifting vibratory stimuli. This training of vibratory stimulation in the lumbar region may have made the region more sensitive to the dynamic movements of the trunk and the center of gravity line; thus, subjects undergoing lumbar training became better able to control trunk movements.

In the present study, the results of the logistic regression analysis showed an association between correct response rate with stimulation of two sites vertically and the forward and backward displacement values in the LoS test, but equations were only found for total antero-posterior movement of the center of gravity, which is easy to comprehend as movement of the trunk, and vertically paired stimulation of the lumbar region, which had a good rate of correct responses. A number of prior studies have reported that tactile sensation such as touch-pressure sensation and vibratory sensation are important in relation to standing balance and gait performance [28-30]. There are also reports that the reactions to tactile sensation are dominant over reactions to visual and vestibular sensations as reactions for postural control $[31,32]$ . From this, it may be concluded that tactile sensation in the trunk and legs is particularly important for avoiding falls when standing or walking. The high correlation between tactile sensation of the trunk and dynamic center of gravity data in the present study points to the importance of trunk sensory training as training for prevention of falls.

As an area for future investigation, we intend to investigate the effects of vibratory stimulation training on brain function, which will include examination of the superiority of the effects on standing balance of a lumbar training group and a plantar training group, examination of the effects on standing balance of long-term sensory training of 12-14 weeks with a larger sample size, examination of gender differences, and examination of the specifications of the vibratory device, including miniaturization and vibratory stimulation at different frequencies.

\section{CONCLUSIONS}

These results suggested that a trunk training may contribute to improved dynamic balance performance. It may be concluded that tactile sensation in the trunk is particularly important for avoiding falls when standing or walking.

\section{ACKNOWLEDGMENT}

The authors would like to thank all subjects who kindly participated in the study.

\section{FUNDING}

This work was supported by Northern Advancement Center for Science and Technology and JSPS KAKENHI Grant Number JP $20 \mathrm{H} 04049$.

\section{REFERENCES}

[1] American Geriatrics Society, British Geriatrics Society, and American Academy of Orthopaedic Surgeons Panel on Falls Prevention: Guideline for the prevention of falls in older persons. Am. Geriatr Soc., 2001; 49 :664-672. [2] A J Blake, K Morgan, M J Bendall, H Dallosso, S B Ebrahim, T H Arie, P H Fentem, E J Bassey. Falls by elderly people at home: prevalence and associated factors. Age and Ageing. 1988;17:365-372.

[3] Yoshida H, Hunkyung K. Frequency of falls and their prevention. Clin calcium. 2006; 16:1444-1450.

[4] Birmingham T. Test-retest reliability of lower extremity functional instability measures. Clin J Sport Med. 2000; 10:264-268.

[5] Brouwer B, Culham EG, Liston RA, Grant T. Normal variability of postural measures: implications for the reliability of relative balance performance outcomes. Scand J Rehabil Med.1998; 30:131-137.

[6] Yusuke Maeda, Toshiaki Tanaka, Tomoya Miyasaka, Koichi Shimizu. A Preliminary Study of Static and Dynamic Standing Balance and Risk of Falling in an Independent Elderly Population with a Particular Focus on the Limit of Stability Test. Journal of Physical Therapy Science. 2011; 23: 803-806.

[7] Bronstein AM, Brandt T, Woollacott M, Nutt JG. Clinical disorders of balance,

posture and gait, 2nd ed., Cary, NC: A Hodder Arnold Publication; 2004.

[8] T. Tanaka, S. Noriyasu, S. Ino, T. Ifukube, M. Nakata : Objective method to determine the contribution of the great toe to standing balance and preliminary observations of age-related effects, IEEE Trans. Rehab. Eng., 1996; 4(2): 84-90.

[9] Inglis JT, Horak FB, Shupert CL, Jones-Rycewicz C. The importance of somatosensory information in triggering and scaling automatic postural responses in

humans. Exp Brain Res 1994; 101:159-64

[10] Kenshalo DR. Somesthetic sensitivity in young and elderly humans. J Gerontol. 1986; 41:732-42.

[11] Tanaka T, Shirogane S, Izumi T, Ino S, and Ifukube T. The Effect of Brief Moving Vibratory Stimulation on the Feet for Postural Control in a Comparison Study. Physical \& Occupational Therapy in Geriatrics, 2005; 24 (1):1-23.

[12] Toshiaki Tanaka, Norio Kato, Yasuhiro Nakajima, Takashi Izumi.Development of a Novel Testing and Training Device of Proprioceptive Sensory for Standing Balance Ability in the Elderly and Disabled Individuals. International Journal of New Technology and Research (IJNTR), 2018; 4(7): p.15-p.20.

[13] Pyykko I, Jantti P, Aalto H. Postural control in elderly subjects. Age Ageing 
1990;19:215-21

[14] LeVeau BF. Williams and Lissner's Biomechanics of human motion. 3rd ed. Philadelphia: WB Saunders. pp12-14,1992.

[15] Jijimol G, Fayaz RK, Vijesh PV. Correlation of trunk impairment with balance in patients with chronic stroke.NeuroRehabilitation. 2013; 32: 323-325.

[16] Johansson G, Jarnlo G. Balance training in 70-year-old women. Physiotherapy Theory and Practice, 1991; 7(2):121-125.

[17] Dean CM, Channon EF, Hall JM. Sitting training early after stroke improves sitting ability and quality and carries over to standing up but not to walking: a randomized trial. Aust J Physiother. 2007; 53(2):97-102.

[18] Gillespie LD., Gillespie WJ., Robertson MC., Lamb SE., Cumming RG., Rowe BH. Interventions for preventing falls in elderly people. Cochrane Database Syst. Rev., 2003; (4): CD 000340.

[19] Buchner DM., Cress ME., de Lateur BJ., Esselman PC., Margherita AJ., Price R. et al.: The effect of strength and endurance training on gait, balance, fall risk, and health services use in community-living older adults. Journals of Gerontology. Series A, Biological Sciences and Medical Sciences, 1997; 52 (4): M 218-224.

[20] Lord SR., Ward JA.,Williams P., Strudwick M. The effect of a 12-month exercise trial on balance,strength, and falls in older women: a randomized controlled trial. Journal of the American Geriatrics Society, 1995; 43 :1198-1206.

[21] HoweTE., Rochester L., Jackson A., Banks PM., Blair,VA. Exercise for improving balance in older people.Cochrane Database Syst.Rev., 2007; (4) :CD 004963

[22] Juras G, Słomka1 K, Fredyk A, Sobota G, Bacik B. Evaluation of the Limits of Stability (LOS) Balance Test. Journal of Human Kinetics. 2008; 19: 39-52.

[23] Whanger A, Wang HS. Clinical correlates of the vibratory sense in elderly psychiatric patients. J gerontology. 1974; 29:39-45.

[24] Johansson R .S .and Vallbo A .B . Tactile sensory coding in the glabrous skin of the human hand. Trends in Neuroscience, 1983; 6:27-32.

[25] S. Ino, S. Shimizu, F. Hosoe, T. Izumi, M. Takahashi and T. Ifukube: A Basic Study on Tactile Display for Tele-presence, IEEE International Workshop on Robot and Human Communication, RO-MAN'92, 1992; 58-62. [26] Priscila G. Franco, 1 Karini B. Santos, 1 and André L. F. Rodacki. Joint positioning sense, perceived force level and two-point discrimination tests of young and active elderly adults. Braz J Phys Ther. 2015; 19(4): 304-310.

[27] Maurer, C., Mergner, T., Bolha, B., \& Hlavacka, F. Human balance control during cutaneous stimulation of the plantar soles. Neuroscience Letters, 2001; 302, 45-48.

[28] Hylton B, Menz 1, Stephen R Lord, Rebecca St George, Richard C Fitzpatrick. Walking stability and sensorymotor function in older people with diabetic peripheral neuropathy. Arch Phys Med Rehabil. 2004; 85:245-252. [29] SR Lord, RD Clark, IW Webster. Postural stability and associated physiological factors in a population of aged persons. J Gerontol. 1991; 46:M69-76

[30] Kavounoudias A, Roll R, Roll J-P. Foot sole and ankle muscle inputs contribute

jointly to human erect posture regulation. J Physiol. 2001; 532:869-78.

[31] Dietz v. Trippel M. Horstmann GA. Significance of proprioceptive and vcstibulo - spinal reflexes in the control of stance and gait. In: Patla AE, ed. Adaptability of human gait. Amsterdam: Elsevier, 1991;17- 52.

[32] Massion J, Wollacott M. Normal balance and postural control. In: Bronstein AM, Brandit, T, Woollacott M. Clinical aspects of balance and gait disorders. London: Edward Arnold; 1996. 